\title{
Nonlinear mathematical model and numerical algorithm for monitoring and predicting the concentration of harmful substances in the atmosphere
}

\author{
Tursun Shafiev $^{1^{*}}$, Gulchera Shadmanova ${ }^{2}$, Khabiba Karimova $^{2}$ and Farrukh Muradov ${ }^{2,3}$ \\ ${ }^{1}$ Bukhara State University, Bukhara, Uzbekistan, \\ ${ }^{2}$ Tashkent Institute of Irrigation and Agricultural Mechanization Engineers, Tashkent, Uzbekistan, \\ ${ }^{3}$ Tashkent pediatric medical institute, Tashkent, Uzbekistan,
}

\begin{abstract}
The article developed a nonlinear mathematical model and an effective numerical algorithm for monitoring and forecasting the process of spreading industrial emissions in the atmosphere, taking into account the orography of the area and the speed of movement of fine substances. Multidimensional partial differential equations describe the model with corresponding initial, internal, and boundary conditions of the third kind to consider mass transfer across the interface. In the work, to prove the adequacy of the developed mathematical apparatus, the calculated data are compared with field data taken from a specific object, in which the computational algorithm provides sufficient accuracy of the solution, based on which it can be concluded that the developed mathematical model and the numerical algorithm have a certain advantage over others numerical methods.
\end{abstract}

\section{Introduction}

Today, air pollution is a global "environmental problem", and mathematical modeling can claim the role of "solution" to these problems and making managerial decisions because mathematical modeling is an effective tool for assessing and analyzing air quality and protecting them from pollutants. From the analysis of the conducted long-term observations, it follows that no emission reduction and control strategy can be economically effective without serious preliminary application of mathematical modeling methods for the above problem. Mathematical modeling is one of the effective practical tools that can answer "what if" questions.

In recent years, scientists have developed mathematical tools for researching, predicting and monitoring the ecological state of industrial regions, which are based on a mathematical model, a numerical algorithm and software for computing experiments on a

* Corresponding author: tursunshafiev@gmail.com 
computer and have obtained significant theoretical and applied results on the above problem.

In particular, in the article of the authors [1], a mathematical model for calculating the concentration of harmful substances in the atmosphere of the city is proposed. The proposed mathematical model is based on a system of transfer equations of a universal form. The basic three-dimensional system of equations in the form of a universal transport equation is investigated based on mathematical models proposed by M.E. Berland [2] and G.I. Marchuk [3]. This research approach allowed the authors to simplify the modeling process by using universal numerical algorithms for all system equations.

In work [4], a methodology is proposed for modeling and optimal control of the hazard of atmospheric pollution in nuclear and chemical disasters. To predict atmospheric pollution during nuclear and chemical disasters, atmospheric dispersion models and explosion hazard assessment models were used, and with the help of these models, a special computational algorithm and an application for modeling gas dispersion were built. The results of model and experimental data are investigated. The meteorological data were modeled using the mesoscale weather forecasting model MESO, and the concentration distribution was modeled using the CDM (Atmospheric Pollution Chemical Accident Hazard Predicting) model [5].

The article [6] discusses the numerical modeling of the process of dispersion of atmospheric pollutants, taking into account the deposition rate of particles. The process model is described by partial differential equations with appropriate initial and boundary conditions. When constructing the model, the basic laws of hydrodynamics were used and a set of parameters that significantly affect the process: weather conditions, the ability to absorb the atmosphere, properties of particles, etc. An equation for calculating the deposition rate of solid spherical particles was developed. For the numerical solution of the problem, an implicit finite-difference scheme in time with the second-order accuracy was used.

The article [7] considers the possibilities of predicting the concentration of pollutants in the atmosphere using the combined model COSMO-Ru7-ART - the synthesis of the mesoscale non-hydrostatic metrological model COSMO-Ru (Consortium for Small-scale Modeling - Russia) and the atmospheric chemistry model ART [8] ( Aerosols and Trace gases). The COSMO-Ru7-ART model is based on a three-dimensional diffusion equation. To verify the model, the authors carried out a computational experiment to calculate the actual emissions of carbon monoxide to refine the forecast of pollution in the territory of Moscow and the Moscow region. It was found that the above model significantly overestimates the predictive concentration of carbon monoxide.

The article [9] developed a mathematical model for the dispersion and transport of pollutants in the atmosphere. The propagation of active and passive impurities from an instantaneous point source in the atmosphere is investigated, and the results of a study of the partial absorption of impurities are proposed. The proposed mathematical model of the problem includes the transport equations, the coefficient of viscosity and turbulence, which take constant values.

In [10], the influence of various typologies of urban areas on the spread of urban pollutants was investigated. Five typologies derived from real cities were investigated using CFD: monoblock, free-standing buildings, central courtyard, courtyards, and row. Numerical simulations were carried out using the URANS (non-stationary ReynoldsNavier-Stokes averaging) equations, and a model was used to describe the turbulence. To check the adequacy of the developed mathematical apparatus, the authors applied it using experimental data in a wind tunnel. From the results obtained, the authors concluded that the higher the wind speed, the lower the concentration of pollutants. Particular attention is 
paid to the location of undeveloped areas because these areas were more important for the dispersion of pollutants.

The authors' article [11] proposes a method for calculating the pollution of industrial centers of the city of Voronezh with heavy metals, taking into account the influence of specific industrial facilities on a local and regional scale. An assessment of the concentration of pollutants in the air and snow in the immediate vicinity of an industrial source of pollutants at a distance of $100 \mathrm{~km}$ has been carried out. The above results show that the proposed model can be successfully used to calculate long-term average concentrations from industrial sources, taking into account the local, regional emissions component.

Practice shows that for a sufficiently accurate prediction of the process of dispersion of pollutants in the atmosphere, it is necessary to take into account in mathematical models such factors as the change in the rate of aerosol released into the atmosphere in three directions, the change in the diffusion coefficient and the coefficient of turbulent mixing with stable and unstable stratification, the characteristics of the wind rose, and orography of the area, the phase transition of substances arising from changes in temperature in the atmosphere. In the work of the authors [12], a mathematical model is considered that takes into account these factors and is focused on a short-term forecast of the concentration of pollutants in the boundary layer of the atmosphere in industrial regions. The developed mathematical model is based on the law of conservation of mass and momentum and is described by the transport and diffusion equations. The authors also developed a numerical algorithm and software for conducting computational experiments. The model was validated based on a short-term prediction of particulate matter concentration emitted from an existing cement plant in the Samarkand region of Uzbekistan.

The development of software products and the automation of industrial enterprises, particularly automation for the stages of continuous production, is associated with a clearly defined practical goal - with the creation of information monitoring systems and systems that facilitate decision-making. The authors [13] use information monitoring and decisionmaking, design, improvement, design and implementation of software systems, data mining, methods of building a client-server architecture, relational data management systems and technologies and methods of object-oriented programming MVC, ORM.

The article of the authors [14] is devoted to air quality modeling based on GIS technology. This technology implies the integration of heterogeneous data and web services into an autonomous modeling system. To study the process under consideration, a mathematical model was developed that considers such important parameters as the rate of particle deposition, the absorption coefficient and the coefficient of interaction with the earth's surface. Based on the results obtained, the authors proposed the optimal placement of newly designed facilities in industrial regions. And also, the scale of the spread of harmful emissions was assessed, followed by making decisions to minimize environmental risks.

In the article of the authors [15], an effective tool was developed for transforming the results obtained using the integrated approach of computational fluid dynamics [16] and computational dynamics of reactions [17]. The most commonly used visualization techniques are being revised and implemented into the transformation tool and their visualization in Google Earth. The potential of this tool is demonstrated by the example of smog generated by heavy vehicle emissions in Rotterdam, The Netherlands. The results showed that Google Earth could provide a computationally efficient and user-friendly presentation of data. The developed function by the authors can be very useful for visualizing street-level pollution, which is of great importance for city residents.

A mathematical model for monitoring, predicting, and assessing the ecological state of the atmosphere and the underlying surface by passive and active impurities, which take into 
account the variable speed of movement of particles in the atmosphere developed in the article [18]. To determine the velocities of movement of fine particles in the atmosphere, a system of nonlinear equations has been developed, which considers the basic physical and mechanical properties of particles and the velocity of air mass in the atmosphere, which plays an important role.

A detailed analysis of scientific works related to the problem of mathematical modeling of the process of transport and diffusion of harmful substances in the atmosphere showed that in the mathematical modeling and research of the process of propagation of harmful substances in the atmosphere in the above works: firstly, the speed of movement of aerosol particles in the atmosphere was not considered as a function of the speeds airflow in the atmosphere, which change over time; secondly, in all the above mathematical models of the process, the orography of the area was not taken into account.

Based on the foregoing, the purpose of this article is to develop a nonlinear mathematical model for monitoring and predicting the process of transport and diffusion of harmful substances in the atmosphere, and the developed mathematical model should take into account the possibility of calculating atmospheric pollution taking into account the orography of the terrain, the speed, and direction of the wind, the speed of mixing particles, as well as the weather and climatic factors.

\section{Methods}

To study the process of transport and diffusion of aerosol particles in the atmosphere, taking into account the essential parameters $u_{u}, v_{u}, w_{u}$, the components of the wind speed in the directions $x, y, z$, respectively, and $h$ the parameter for determining the terrain relief, consider a mathematical model describing the basis of the law of hydromechanics using a multidimensional partial differential equation:

$$
\begin{aligned}
\frac{\partial \theta}{\partial t}+u_{u} \frac{\partial h \theta}{\partial x}+v_{u} \frac{\partial h \theta}{\partial y}+w_{u} \frac{\partial h \theta}{\partial z}+\sigma h \theta=\mu\left(\frac{\partial^{2} h \theta}{\partial x^{2}}+\frac{\partial^{2} h \theta}{\partial y^{2}}\right)+\frac{\partial}{\partial z}\left(\kappa \frac{\partial h \theta}{\partial z}\right)+\delta Q \\
m \frac{d u_{u}}{d t}=c_{f} \pi r^{2} \rho_{6}\left(u_{u}-U\right)^{2} \\
m \frac{d v_{u}}{d t}=c_{f} \pi r^{2} \rho_{\theta}\left(v_{u}-U\right)^{2} \\
m \frac{d w_{u}}{d t}=-\frac{4}{3} \pi r^{3}\left(\rho_{u}-\rho_{6}\right) g-k_{f} \mu_{6} \pi r w_{u}+F_{n}
\end{aligned}
$$

with corresponding initial

$$
\theta(x, y, z, 0)=\theta^{0}(x, y, z), u_{u}=u(0), v_{u}=v(0), w_{u}=w(0), \text { at } \mathrm{t}=0
$$

and boundary conditions

$$
-\left.\mu \frac{\partial h \theta}{\partial x}\right|_{x=0}=\xi h\left(\theta_{\theta}-\theta\right) ;\left.\quad \mu \frac{\partial h \theta}{\partial x}\right|_{x=L_{x}}=\xi h\left(\theta_{\theta}-\theta\right) ;
$$




$$
\begin{aligned}
-\left.\mu \frac{\partial h \theta}{\partial y}\right|_{y=0}=\xi h\left(\theta_{\theta}-\theta\right) ; & \left.\mu \frac{\partial h \theta}{\partial y}\right|_{y=L_{y}}=\xi h\left(\theta_{\theta}-\theta\right) ; \\
-\left.\kappa \frac{\partial h \theta}{\partial z}\right|_{z=0}=\xi h\left(\beta \theta-F_{0}\right) ; & \left.\kappa \frac{\partial \theta h}{\partial z}\right|_{z=H}=\xi h\left(\theta_{\theta}-\theta\right) ;
\end{aligned}
$$

where $U=\sqrt{u^{2}+v^{2}+w^{2}}$.

Here $\mathrm{t}$ is time; $x, y, z$ are coordinates; $\theta$ is the concentration of the spreading substance; $\mathrm{h}$ is parameter for determining the terrain; $\sigma$ is coefficient of absorption of harmful substances in the atmosphere; $\mu$ is the diffusion coefficient; $\mathrm{k}$ is turbulence coefficient; $\delta_{\text {is }}$ Dirac function; $Q$ is power of sources; $\theta^{0}$ is the primary concentration of harmful substances in the atmosphere; $\mathrm{m}$ is the mass of the particle; $c_{f}$ is coefficient of drag of particles; $r$ is the particle radius; $\rho_{b}$ is air density; $\rho_{u}$ is particle density; $g$ is acceleration of free fall; $k_{f}$ is body shape coefficient for the resistance force; $\mu_{b}$ is air viscosity; $F_{n}$ is the lifting force of the air flow; $\beta$ is coefficient of interaction with the underlying surface; $F_{0}$ is the number of aerosol particles detached from the roughness of the earth's surface; $\xi$ is coefficient for carrying out the boundary condition to the dimensional form; $\theta_{6}$ is concentration of suspended solids in neighboring areas of the tasks being solved.

The parameter for determining the terrain is determined using the ratio [19]:

$$
h=\left\{\begin{array}{l}
0-\text { If the layer is underground; } \\
1-\text { If the layer is in the atmosphere; } \\
\frac{\left(\eta-z_{k-0,5}\right)}{\Delta z}-\text { If the layer is below } \\
\text { the orographic surface }
\end{array}\right.
$$

Here $\eta$ is the height of the elevation above the plane parallel to sea level, and $\Delta z=z_{k+0,5}-z_{k-0,5}$. For each layer, the multiplier $h(0 \leq h \leq 1)$, is introduced that determines the degree of blocking of the air flow.

Since problem (1) - (8) is described by a multidimensional nonlinear partial differential equation with the corresponding initial and boundary conditions, it is difficult to obtain its solution in analytical form. To solve the problem, we use an implicit finite-difference scheme in time with the second-order of accuracy, respectively, in, and finally, we obtain a system of algebraic equations to $O x$ [20-24]:

$$
a_{i, j, k} \theta_{i-1, j, k}^{n+1 / 3}-b_{i, j, k} \theta_{i, j, k}^{n+1 / 3}+c_{i, j, k} \theta_{i+1, j, k}^{n+1 / 3}=-d_{i, j, k},
$$

where the sweep coefficients $\alpha_{0, j, k}$ and $\beta_{0, j, k}$ computed with: 


$$
\begin{aligned}
& a_{i, j, k}=\frac{\mu h_{i-0,5, j, k}}{\Delta x^{2}}+\frac{u_{u}^{n+1 / 3}+\left|u_{u}^{n+1 / 3}\right|}{4 \Delta x} h_{i-0,5, j, k} ; \quad b_{i, j, k}= \\
& =\frac{\mu h_{i+0,5, j, k}+\mu h_{i-0,5, j, k}}{\Delta x^{2}}+\frac{\left|u_{\psi}^{n+1 / 3}\right| h_{i, j, k}}{2 \Delta x}+\frac{3}{2 \Delta t}+h_{i, j, k} \sigma ; \\
& c_{i, j, k}=\frac{\mu h_{i+0,5, j, k}}{\Delta x^{2}}-\frac{u_{u}^{n+1 / 3}-\left|u_{u}^{n+1 / 3}\right|}{4 \Delta x} h_{i+0,5, j, k}-\frac{3}{2 \Delta t} \text {; } \\
& d_{i, j, k}=\left(\frac{3}{2 \Delta t}-\frac{h_{i, j+0,5, k}+h_{i, j-0,5, k}}{\Delta y^{2}} \mu-\right. \\
& \left.-\frac{\kappa_{k+0,5} h_{i, j, k+0,5}+\kappa_{k-0,5} h_{i, j, k-0,5}}{\Delta z^{2}}-\frac{\left|u_{u}^{n+1 / 3}\right| h_{i, j, k}}{2 \Delta x}-\frac{\left|v_{u}^{n+1 / 3}\right| h_{i, j, k}}{\Delta y}-\frac{\left|w_{u}^{n+1 / 3}\right| h_{i, j, k}}{\Delta z}\right) \theta_{i, j, k}^{n}+ \\
& +\left(\frac{u_{u}^{n+1 / 3}+\left|u_{u}^{n+1 / 3}\right|}{4 \Delta x} h_{i-0,5, j, k}\right) \theta_{i-1, j, k}^{n}+\left(\frac{3}{2 \Delta t}-\frac{u_{u}^{n+1 / 3}-\left|u_{u}^{n+1 / 3}\right|}{4 \Delta x} h_{i+0,5, j, k}\right) \theta_{i+1, j, k}^{n}+ \\
& +\left(\frac{\mu}{\Delta y^{2}}+\frac{v_{u}^{n+1 / 3}+\left|v_{u}^{n+1 / 3}\right|}{2 \Delta y}\right) h_{i, j-0,5, k} \theta_{i, j-1, k}^{n}+\left(\frac{\mu}{\Delta y^{2}}-\frac{v_{u}^{n+1 / 3}-\left|v_{u}^{n+1 / 3}\right|}{2 \Delta y}\right) h_{i, j+0,5, k} \theta_{i, j+1, k}^{n}+ \\
& +\left(\frac{\kappa_{k-0,5}}{\Delta z^{2}}+\frac{w_{u}^{n+1 / 3}+\left|w_{u}^{n+1 / 3}\right|}{2 \Delta z}\right) h_{i, j, k-0,5} \theta_{i, j, k-1}^{n}+ \\
& +\left(\frac{\kappa_{k+0,5}}{\Delta z^{2}}-\frac{w_{u}^{n+1 / 3}-\left|w_{u}^{n+1 / 3}\right|}{2 \Delta z}\right) h_{i, j, k+0,5} \theta_{i, j, k+1}^{n}+\frac{1}{3} \delta_{i, j, k} Q .
\end{aligned}
$$

Approximating the boundary conditions (6) at, opening parentheses and grouping similar terms of the equation, we obtain the values $\theta_{0, j, k}^{n+1 / 3}$ :

$$
\begin{aligned}
& \theta_{0, j, k}^{n+1 / 3}=\frac{4 \mu c_{1, j, k} h_{0,5, j, k}-b_{1, j, k} \mu h_{1, j, k}}{3 \mu c_{1, j, k} h_{0, j, k}-a_{1, j, k} \mu h_{1, j, k}+2 \Delta x \xi c_{1, j, k} h_{0, j, k}} \theta_{1, j, k}^{n+1 / 3}+ \\
& +\frac{d_{1, j, k} \mu h_{1, j, k}+2 \Delta x \xi c_{1, j, k} h_{0, j, k} \theta_{E}}{3 \mu c_{1, j, k} h_{0, j, k}-a_{1, j, k} \mu h_{1, j, k}+2 \Delta x \xi c_{1, j, k} h_{0, j, k}}
\end{aligned}
$$

где $\alpha_{0, j, k}$ and $\beta_{0, j, k}$ are defined as follows:

$$
\begin{aligned}
& \alpha_{0, j, k}=\frac{4 \mu c_{1, j, k} h_{0,5, j, k}-b_{1, j, k} \mu h_{1, j, k}}{3 \mu c_{1, j, k} h_{0, j, k}-a_{1, j, k} \mu h_{1, j, k}+2 \Delta x \xi c_{1, j, k} h_{0, j, k}} ; \\
& \beta_{0, j, k}=\frac{d_{1, j, k} \mu h_{1, j, k}+2 \Delta x \xi c_{1, j, k} h_{0, j, k} \theta_{6}}{3 \mu c_{1, j, k} h_{0, j, k}-a_{1, j, k} \mu h_{1, j, k}+2 \Delta x \xi c_{1, j, k} h_{0, j, k}} .
\end{aligned}
$$


Further, approximating the boundary conditions (6) at, opening parentheses, grouping similar terms of the equation, we obtain the values $\theta_{N, j, k}^{n+1 / 3}$ :

$$
\theta_{N, j, k}^{n+1 / 3}=\frac{2 \Delta x \xi h_{N, j, k} \theta_{B}-\left(\alpha_{N-2, j, k} \beta_{N-1, j, k} h_{N-1, j, k}+\beta_{N-2, j, k} h_{N-1, j, k}-4 \beta_{N-1, j, k} h_{N-0,5, j, k}\right) \mu}{\left(\alpha_{N-2, j, k} \alpha_{N-1, j, k} h_{N-1, j, k}-4 \alpha_{N-1, j, k} h_{N-0,5, j, k}+3 h_{N, j, k}\right) \mu+2 \Delta x \xi h_{N, j, k}} .
$$

Concentration sequence values $\theta_{N-1, j, k}^{n+\frac{1}{3}}, \theta_{N-2, j, k}^{n+\frac{1}{3}}, \ldots, \theta_{1, j, k}^{n+\frac{1}{3}}$ determined by method reverse run.

Similarly, using the above technology by coordinate $O y$ and get:

$$
\bar{a}_{i, j, k} \theta_{i, j-1, k}^{n+2 / 3}-\bar{b}_{i, j, k} \theta_{i, j, k}^{n+2 / 3}+\bar{c}_{i, j, k} \theta_{i, j+1, k}^{n+2 / 3}=-\bar{d}_{i, j, k},
$$

where the elements of the transfer matrix are calculated using:

$$
\begin{aligned}
& \bar{a}_{i, j, k}=\frac{\mu h_{i, j-0,5, k}}{\Delta y^{2}}+\frac{v_{u}^{n+2 / 3}+\left|v_{u}^{n+2 / 3}\right|}{4 \Delta y} h_{i, j-0,5, k} \\
& \bar{b}_{i, j, k}=\frac{\mu h_{i, j+0,5, k}+\mu h_{i, j-0,5, k}}{\Delta y^{2}}+\frac{\left|v_{u}^{n+2 / 3}\right| h_{i, j, k}}{2 \Delta y}+\frac{3}{2 \Delta t}+h_{i, j, k} \sigma \\
& \bar{c}_{i, j, k}=\frac{\mu h_{i, j+0,5, k}}{\Delta y^{2}}-\frac{v_{u}^{n+2 / 3}-\left|v_{u}^{n+2 / 3}\right|}{4 \Delta y} h_{i, j, k+0,5}-\frac{3}{2 \Delta t} \\
& \bar{d}_{i, j, k}=\left(\frac{3}{2 \Delta t}-\frac{\mu h_{i+0,5, j, k}+\mu h_{i-0,5, j, k}}{\Delta x^{2}}-\right. \\
& \left.-\frac{\kappa_{k+0,5} h_{i, j, k+0,5}+\kappa_{k-0,5} h_{i, j, k-0,5}}{\Delta z^{2}}-\frac{\left|u_{u}^{n+2 / 3}\right| h_{i, j, k}}{\Delta x}-\frac{\left|v_{u}^{n+2 / 3}\right| h_{i, j, k}}{2 \Delta y}-\frac{\left|w_{u}^{n+2 / 3}\right| h_{i, j, k}}{\Delta z}\right) \theta_{i, j, k}^{n+1 / 3}+ \\
& +\left(\frac{\mu}{\Delta x^{2}}+\frac{u_{u}^{n+2 / 3}+\left|u_{u}^{n+2 / 3}\right|}{2 \Delta x}\right) h_{i-0,5, j, k} \theta_{i-1, j, k}^{n+1 / 3}+\left(\frac{\mu}{\Delta x^{2}}-\frac{u_{u}^{n+2 / 3}-\left|u_{u}^{n+2 / 3}\right|}{2 \Delta x}\right) h_{i+0,5, j, k} \theta_{i+1, j, k}^{n+1 / 3}+ \\
& +\left(\frac{v_{u}^{n+2 / 3}+\left|v_{u}^{n+2 / 3}\right|}{4 \Delta y} h_{i, j-0,5, k}\right) \theta_{i, j-1, k}^{n+1 / 3}+\left(\frac{3}{2 \Delta t}-\frac{v_{u}^{n+2 / 3}-\left|v_{u}^{n+2 / 3}\right|}{4 \Delta y} h_{i, j+0,5, k}\right) \theta_{i, j+1, k}^{n+1 / 3}+ \\
& +\left(\frac{\kappa_{k-0,5}}{\Delta z^{2}}+\frac{w_{u}^{n+2 / 3}+\left|w_{u}^{n+2 / 3}\right|}{2 \Delta z}\right) h_{i, j, k-0,5} \theta_{i, j, k-1}^{n+1 / 3}+ \\
& +\left(\frac{\kappa_{k+0,5}}{\Delta z^{2}}-\frac{w_{u}^{n+2 / 3}-\left|w_{u}^{n+2 / 3}\right|}{2 \Delta z}\right) h_{i, j, k+0,5} \theta_{i, j, k+1}^{n+1 / 3}+\frac{1}{3} \delta_{i, j, k} Q .
\end{aligned}
$$


Approximating the boundary conditions (7) at, opening parentheses, grouping similar terms of the equation, we obtain the values $\theta_{i, 0, k}^{n+1 / 3}$ :

$$
\begin{aligned}
& \theta_{i, 0, k}^{n+2 / 3}=\frac{4 \mu \bar{c}_{i, 1, k} h_{i, 0,5, k}-\bar{b}_{i, 1, k} \mu h_{i, 1, k}}{3 \mu \bar{c}_{i, 1, k} h_{i, 0, k}-\bar{a}_{i, 1, k} \mu h_{i, 1, k}+2 \Delta y \xi \bar{c}_{i, 1, k} h_{i, 0, k}} \theta_{i, 1, k}^{n+2 / 3}+ \\
& +\frac{\bar{d}_{i, 1, k} \mu h_{i, 1, k}+2 \Delta y \xi \bar{c}_{i, 1, k} h_{i, 0, k} \theta_{b}}{3 \mu \bar{c}_{i, 1, k} h_{i, 0, k}-\bar{a}_{i, 1, k} \mu h_{i, 1, k}+2 \Delta y \xi \bar{c}_{i, 1, k} h_{i, 0, k}}
\end{aligned}
$$

where race coefficients $\bar{\alpha}_{i, 0, k}$ and $\bar{\beta}_{i, 0, k}$ are calculated using:

$$
\begin{aligned}
& \bar{\alpha}_{i, 0, k}=\frac{4 \mu \bar{c}_{i, 1, k} h_{i, 0,5, k}-\bar{b}_{i, 1, k} \mu h_{i, 1, k}}{3 \mu \bar{c}_{i, 1, k} h_{i, 0, k}-\bar{a}_{i, 1, k} \mu h_{i, 1, k}+2 \Delta y \xi \bar{c}_{i, 1, k} h_{i, 0, k}} ; \bar{\beta}_{i, 0, k}= \\
& =\frac{\bar{d}_{i, 1, k} \mu h_{i, 1, k}+2 \Delta y \xi \bar{c}_{i, 1, k} h_{i, 0, k} \theta_{b}}{3 \mu \bar{c}_{i, 1, k} h_{i, 0, k}-\bar{a}_{i, 1, k} \mu h_{i, 1, k}+2 \Delta y \xi \bar{c}_{i, 1, k} h_{i, 0, k}} .
\end{aligned}
$$

Further, approximating the boundary condition (7) at $y=L_{y}$, opening the brackets, grouping similar terms of the equation, we get the values $\theta_{i, M, k}^{n+2 / 3}$ :

$$
\theta_{i, M, k}^{n+2 / 3}=\frac{2 \Delta y \xi h_{i, M, k} \theta_{\theta}-\left(\bar{\alpha}_{i, M-2, k} \bar{\beta}_{i, M-1, k} h_{i, M-1, k}+\bar{\beta}_{i, M-2, k} h_{i, M-1, k}-4 \bar{\beta}_{i, M-1, k} h_{i, M-0,5, k}\right) \mu}{\left(\bar{\alpha}_{i, M-2, k} \bar{\alpha}_{i, M-1, k} h_{i, M-1, k}-4 \bar{\alpha}_{i, M-1, k} h_{i, M-0,5, k}+3 h_{i, M, k}\right) \mu+2 \Delta y \xi h_{i, M, k}}
$$

Concentration sequence values $\theta_{i, M-1, k}^{n+\frac{2}{3}} \theta_{i, M-2, k}^{n+\frac{2}{3}}, \ldots, \theta_{i, 1, k}^{n+\frac{2}{3}}$ determined by the reverse sweep method.

Similarly, using the above technology by coordinate $\mathrm{Oz}$ and get:

$$
\overline{\bar{a}}_{i, j, k} \theta_{i, j, k-1}^{n+1}-\overline{\bar{b}}_{i, j, k} \theta_{3, i, j, k}^{n+1}+\overline{\bar{c}}_{i, j, k} \theta_{i, j, k+1}^{n+1}=-\overline{\bar{d}}_{i, j, k},
$$

where the elements of the transfer matrix are calculated:

$$
\begin{aligned}
\overline{\bar{a}}_{i, j, k}= & \frac{\kappa_{k-0,5} h_{i, j, k-0,5}}{\Delta z^{2}}+\frac{w_{u}^{n+1}+\left|w_{u}^{n+1}\right|}{4 \Delta z} h_{i, j, k-0,5} ; \\
\overline{\bar{b}}_{i, j, k}= & \frac{\kappa_{k-0,5} h_{i, j, k-0,5}+\kappa_{k+0,5} h_{i, j, k+0,5}}{\Delta z^{2}}+\frac{\left|w_{u}^{n+1}\right| h_{i, j, k}}{2 \Delta z}+\frac{3}{2 \Delta t}+h_{i, j, k} \sigma ; \\
& \overline{\bar{c}}_{i, j, k}=\frac{\kappa_{k+0,5} h_{i, j, k+0,5}}{\Delta z^{2}}-\frac{w_{u}^{n+1}-\left|w_{u}^{n+1}\right|}{4 \Delta z} h_{i, j, k+0,5}-\frac{3}{2 \Delta t} ;
\end{aligned}
$$




$$
\begin{gathered}
\overline{\bar{d}}_{i, j, k}=\left(\frac{3}{2 \Delta t}-\frac{h_{i+0,5, j, k}+h_{i-0,5, j, k}}{\Delta x^{2}} \mu-\right. \\
\left.-\frac{h_{i, j+0,5, k}+h_{i, j-0,5, k}}{\Delta y^{2}} \mu-\frac{\left|u_{u}^{n+1}\right| h_{i, j, k}}{\Delta x}-\frac{\left|v_{u}^{n+1}\right| h_{i, j, k}}{\Delta y}-\frac{\left|w_{u}^{n+1}\right| h_{i, j, k}}{2 \Delta z}\right) \theta_{i, j, k}^{n+2 / 3}+ \\
+\left(\frac{\mu}{\Delta x^{2}}+\frac{u_{u}^{n+1}+\left|u_{u}^{n+1}\right|}{2 \Delta x}\right) h_{i-0,5, j, k} \theta_{i-1, j, k}^{n+2 / 3}+\left(\frac{\mu}{\Delta x^{2}}-\frac{u_{u}^{n+1}-\left|u_{u}^{n+1}\right|}{2 \Delta x}\right) h_{i+0,5, j, k} \theta_{i+1, j, k}^{n+2 / 3}+ \\
+\left(\frac{\mu}{\Delta y^{2}}+\frac{v_{u}^{n+1}+\left|v_{u}^{n+1}\right|}{2 \Delta y}\right) h_{i, j-0,5, k} \theta_{i, j-1, k}^{n+2 / 3}+\left(\frac{\mu}{\Delta y^{2}}-\frac{v_{u}^{n+1}-\left|v_{u}^{n+1}\right|}{2 \Delta y}\right) h_{i, j+0,5, k} \theta_{i, j+1, k}^{n+2 / 3}+ \\
+\left(\frac{w_{u}^{n+1}+\left|w_{u}^{n+1}\right|}{4 \Delta z} h_{i, j, k-0,5}\right) \theta_{i, j-1, k}^{n+2 / 3}+\left(\frac{3}{2 \Delta t}-\frac{w_{u}^{n+1}-\left|w_{u}^{n+1}\right|}{4 \Delta z} h_{i, j, k+0,5}\right) \theta_{i, j+1, k}^{n+2 / 3}+ \\
+\frac{1}{3} \delta_{i, j, k} Q .
\end{gathered}
$$

Approximating the boundary conditions (8) at $z=0$, opening the parentheses, grouping similar terms of the equation, we obtain the values $\theta_{i, j, 0}^{n+1}$ :

$$
\theta_{i, j, 0}^{n+1}=\frac{4 \kappa_{1} \overline{\bar{c}}_{1} h_{i, j, 0,5}-\overline{\bar{b}}_{1} \kappa_{1} h_{i, j, 1}}{3 \kappa_{1} \overline{\bar{c}}_{1} h_{i, j, 0}-\overline{\bar{a}}_{1} \kappa_{1} h_{i, j, 1}-2 \Delta z \beta \overline{\bar{c}}_{1} h_{i, j, 0}} \theta_{i, j, 1}^{n+1}+\frac{\overline{\bar{d}}_{1} \kappa_{1} h_{i, j, 1}-2 \Delta z \overline{\bar{c}}_{1} h_{i, j, 0} F_{0}}{3 \kappa_{1} \overline{\bar{c}}_{1} h_{i, j, 0}-\overline{\bar{a}}_{1} \kappa_{1} h_{i, j, 1}-2 \Delta z \beta \overline{\overline{c_{1}}} h_{i, j, 0}} ;
$$

where the sweep factors $\overline{\bar{\alpha}}_{i, j, 0}$ and $\overline{\bar{\beta}}_{i, j, 0}$ are calculated using:

$$
\begin{gathered}
\overline{\bar{\alpha}}_{i, j, 0}=\frac{4 \kappa_{1} \overline{\bar{c}}_{1} h_{i, j, 0,5}-\overline{\bar{b}}_{1} \kappa_{1} h_{i, j, 1}}{3 \kappa_{1} \overline{\bar{c}}_{1} h_{i, j, 0}-\overline{\bar{a}}_{1} \kappa_{1} h_{i, j, 1}-2 \Delta z \beta \overline{\bar{c}}_{1} h_{i, j, 0}} ; \\
\overline{\bar{\beta}}_{i, j, 0}=\frac{\overline{\bar{d}}_{1} \kappa_{1} h_{i, j, 1}-2 \Delta z \overline{\bar{c}}_{1} h_{i, j, 0} F_{0}}{3 \kappa_{1} \overline{\bar{c}}_{1} h_{i, j, 0}-\overline{\bar{a}}_{1} \kappa_{1} h_{i, j, 1}-2 \Delta z \beta \overline{\bar{c}}_{1} h_{i, j, 0}} .
\end{gathered}
$$

Approximating the boundary conditions (8) at $z=L_{z}$ opening parentheses, grouping similar terms of the equation, we obtain the values $\theta_{i, j, 0}^{n+1}$ :

$$
\theta_{i, j, L}^{n+1}=\frac{2 \Delta z \xi h_{i, j, L} \theta_{\theta}-\left(\overline{\bar{\alpha}}_{i, j, L-2} \overline{\bar{\beta}}_{i, j, L-1} h_{i, j, L-1}+\overline{\bar{\beta}}_{i, j, L-2} h_{i, j, L-1}-4 \overline{\bar{\beta}}_{i, j, L-1} h_{i, j, L-0,5}\right) \mu}{\left(\overline{\bar{\alpha}}_{i, j, L-2} \overline{\bar{\alpha}}_{i, j, L-1} h_{i, j, L-1}-4 \overline{\bar{\alpha}}_{i, j, L-1} h_{i, j, L-0,5}+3 h_{i, j, L}\right) \mu+2 \Delta z \xi h_{i, j, L}}
$$

To solve equation (2) - (4), we use the implicit scheme [21-23]:

$$
u_{u}^{n+\frac{1}{3}}=\frac{3 m}{3 m-2 c_{f} \pi r^{2} \rho_{\theta} \tilde{u} \Delta t+2 c_{f} \pi r^{2} \rho_{\theta} U \Delta t} u_{u}^{n}-\frac{c_{f} \pi r^{2} \rho_{s} \tilde{u}^{2} \Delta t+c_{f} \pi r^{2} \rho_{\theta} U^{2} \Delta t}{3 m-2 c_{f} \pi r^{2} \rho_{\theta} \tilde{u} \Delta t+2 c_{f} \pi r^{2} \rho_{\theta} U \Delta t} \text {. }
$$




$$
\begin{gathered}
v_{u}{ }^{n+\frac{1}{3}}=\frac{3 m}{3 m-2 c_{f} \pi r^{2} \rho_{\theta} \tilde{v} \Delta t+2 c_{f} \pi r^{2} \rho_{\theta} U \Delta t} v_{u}^{n}-\frac{c_{f} \pi r^{2} \rho_{b} \tilde{v}^{2} \Delta t+c_{f} \pi r^{2} \rho_{b} U^{2} \Delta t}{3 m-2 c_{f} \pi r^{2} \rho_{\theta} \tilde{v} \Delta t+2 c_{f} \pi r^{2} \rho_{\theta} U \Delta t} ; \\
w_{u}{ }^{n+\frac{1}{3}}=\frac{9 m}{9 m+3 k_{f} \mu_{6} \pi r \Delta t} w_{g}^{n}-\frac{4 \pi r^{3}\left(\rho_{n}-\rho_{\theta}\right) g \Delta t-3 F_{n} \Delta t}{9 m+3 k_{f} \mu_{s} \pi r \Delta t} .
\end{gathered}
$$

Similarly, using the above technology, the value $u_{u}^{n+\frac{2}{3}}, u_{u}^{n+1}, v_{u}{ }^{n+\frac{2}{3}}, v_{u}^{n+1}, w_{u}{ }^{n+\frac{2}{3}}, w_{u}^{n+1}$ is calculated at each time layer. The convergence of the iterative process is checked using the conditions:

$$
\left|u^{(s+1)}-u^{(s)}\right|<\varepsilon ;\left|v^{(s+1)}-v^{(s)}\right|<\varepsilon ;\left|w_{g}^{(s+1)}-w_{g}^{(s)}\right|<\varepsilon ;
$$

here $\varepsilon$ is the required accuracy of the solution, $S$ is the number of iterations, while the initial iterative value is chosen equal to the solution at the previous time level $[24,25]$.

\section{Result and Discussion}

Developed a mathematical model of the process of transport and diffusion of harmful substances in the atmosphere, which special attention is paid to determining the speed of the considered harmful substances, based on the physical and mechanical properties of particles, also takes into account such parameters as orography of the area under consideration.

A numerical algorithm has been developed to solve the problem using the approximation of a finite difference scheme. The obtained computational algorithm has the second-order of technicality in time and spatial variables.

To determine the area's relief, a functional dependence is proposed, which determines the coefficient of the orography of the area at each time layer.

Based on the proposed model, it is possible to study the transfer process of the diffusion of harmful substances in the atmosphere. Using the mathematical apparatus, it is possible to predict the optimal location of newly created industrial facilities and estimate the scale of emissions of harmful substances from anthropogenic sources. Using this mathematical model and a computational algorithm to create software for a computer, then using this software it is possible to estimate the concentration of harmful substances in the atmosphere, followed by making decisions to minimize the risks of violation of sanitary standards for the environment.

\section{Conculion}

There was a developed software tool based on the developed mathematical model and numerical algorithm. This software tool can be used to solve practical problems related to monitoring and forecasting the ecological conditions of the atmosphere over industrial regions. It is possible to assess the impact of each type of air pollutant on the ecological conditions using the software tool, taking into account the standards of maximum permissible concentrations. 


\section{References}

1. Sukhinov A.I, Gadelshin V.K and Lyubomishchenko J.S, Mathematical modeling of the spread of harmful impurities in the city atmosphere based on the iocv method, Izvestia SFedU. Engineering pp 177-86, (2005)

2. Berlyand M E, Forecast and regulation of air pollution. L: Gidrometeoizdat. (1985).

3. Marchuk G. I, Mathematical modeling in the environmental problem. M: Nauka. (1982).

4. Shunxiang H, Feng L, Qingcun Z, Fei H and Zifa W, Modeling and Optimal Control of Atmospheric Pollution Hazard in Nuclear and Chemical Disasters UTAM Symposium on the Dynamics of Extreme Events Influenced by Climate Change. Procedia IUTAM 17. pp 79-90. (2015).

5. HUANG S, CHEN H, LIU F, LIU S and ZHU F, Numerical Simulation and Experimental Comparison on Atmospheric Pollution Chemical Accident Hazard Predicting(CDM) Acta Sci. Nat. Univ. Pekin. 47 pp 664-70. (2011).

6. Muradov F and Akhmedov D, Numerical Modeling of Atmospheric Pollutants Dispersion Taking into Account Particles Settling Velocity International Conference on Information Science and Communications Technologies: Applications, Trends and Opportunities, ICISCT 2019. Institute of Electrical and Electronics Engineers Inc. (2019)

7. Surkova G. V, Kirsanov A. A, Kislov A. V, Revokatova A .P and Rivin G. S, Prediction of the concentration of pollutants using the combined model COSMORU7-ART Proceedings of the Hydrometeorological Research Center of the Russian Federation pp 115-38 (2014)

8. Pruppacher H R and Klett J D, The Atmospheric Aerosol and Trace Gases (Springer, Dordrecht) pp 216-86. (2010)

9. Aydosov A, Urmashev B and Zaurbekova G, Modeling the spread of harmful substances in the atmosphere at a variable velocity profile Open Eng. 1 pp 264-269. (2016).

10. Mirzaev S.S., Kholmatova I., Shadmanova G., Yusupov M. and Kubyashev K. Numerical modeling of two-dimensional two-phase filtration under frontal drive. Construction Mechanics, Hydraulics and Water Resources Engineering (CONMECHYDRO - 2020). Tashkent Institute of Irrigation and Agricultural Mechanization Engineers. 23-25 April, (2020).

11. Mirzaev S.S., Tukhtanazarov D., and Karimova Kh.Kh. Software for Determining Residual Oil Reserves in Oil Deposit Development. Construction Mechanics, Hydraulics and Water Resources Engineering (CONMECHYDRO - 2020). Tashkent Institute of Irrigation and Agricultural Mechanization Engineers. 23-25 April, 2020. (2020),

12. Sharipov D, Muradov F and Akhmedov D, Numerical modeling method for shortterm air quality orecast in industrial regions Appl. Math. E - Notes (2019)

13. Mo'minov B B and Eshankulov Kh, Modelling asynchronous parallel process with Petri net Int. J. Eng. Adv. Technol. 8 400-5. (2019)

14. Akhmedov D, Ravshanov N and Muradov F, Mathematical software to study the harmful substances diffusion in the atmosphere PONTE Int. Sci. Res. J. (2018)

15. Liu D and Kenjeres S, Google-earth based visualizations for environmental flows and pollutant dispersion in urban areas Int. J. Environ. Res. Public Health (2017)

16. Thabet S and Thabit T H, Computational Fluid Dynamics: Science of the Future Int. J. Res. Eng. 5 430-3 (2018)

17. Laganà $\mathrm{A}$ and Riganelli $\mathrm{A}$, Computational Reaction and Molecular Dynamics: from Simple Systems and Rigorous Methods to Large Systems and Approximate Methods 
Reaction and Molecular Dynamics. Springer, Berlin, Heidelberg. pp 1-12 (2020)

18. Ravshanov $\mathrm{N}$ and Shafiev $\mathrm{T}$ R, Nonlinear mathematical model for monitoring and predicting the process of transfer and diffusion of fine-dispersed aerosol particles in the atmosphere Journal of Physics: Conference Series. (2019)

19. Sharipov D K, Tashtemirova N and Narzullayeva N, Numerical modeling of the spread of harmful substances in the atmosphere taking into account terrain Probl. Comput. Appl. Math. 1 pp 60-72. (2016)

20. Ravshanov N, Shafiev T R and Tashtemirova N, Nonlinear mathematical model for monitoring and forecasting the process of distributing aerosol particles in the atmosphere Bull. TUIT Manag. Commun. Technol. 1 pp 1-9. (2018).

21. Ravshanov N, Abdullayev Z and Shafiev T R, Mathematical model and numerical algortm to study the process of aerosol particles distribution in the atmosphere. International Conference on Information Science and Communications Technologies (ICISCT) Tashkent: IEEE. (2019)

22. Ravshanov N, Muradov F, Shafiev T. Mathematical model and effective numerical algorithm for monitoring and predicting the concentration of harmful substances in the atmosphere, taking into account the physical and mechanical properties of particles. Problems of Computational and Applied Mathematics 5. pp 1-22. (2020)

23. Shadmanova G., Karimova Kh.Kh, and Ziyaeva Sh.K. Choice of optimal options for land use of farms with the application of information technologies. International Conference on Information Science and Communications Technologies (ICISCT). Tashkent. (2019).

24. Shadmanova G. Karimova Kh.Kh. Information systems in water management. International Conference on Information Science and Communications Technologies (ICISCT). Tashkent. (2019)

25. Shodmonova G., Islomov U., Inamov A., Samadov N. On one approach to numerical solution of nonlinear integro-differential equations. International Conference on Materials Physics, Building Structures and Technologies in Construction, Industrial and Production Engineering (MPCPE-2020). Vladimir, Russia, April 27-28, (2020).

26. Karimova X., Xikmatullaev S., Oymatov R. Vertical nonlinear oscillations of viscoelastic systems with multiple degrees of freedom. International Conference on Materials Physics, Building Structures and Technologies in Construction, Industrial and Production Engineering (MPCPE-2020). Vladimir, Russia, April 27-28, (2020) 